Jpn. J. Phys. Fitness Sports Med. 2006, 55 Suppl. : S1 \$6

\title{
THE EFFECT OF NECK MUSCLE TRAINING ON THE ISOMETRIC CERVICAL EXTENSION STRENGTH AND CROSS-SECTIONAL AREA OF THE NECK EXTENSOR MUSCLES - COMBINED TRAINING FOR NECK EXTENSOR MUSCLES USING A CERVICAL EXTENSION MACHINE-
}

\author{
KAORU TSUYAMA ${ }^{1)}$, YOSUKE YAMAMOTO ${ }^{2)}$, \\ KOICHI NAKAZATO ${ }^{3)}$ and HIROYUKI NAKAJIMA ${ }^{4)}$
}

\begin{abstract}
The purpose of this study was to examine the effect of two kinds of neck muscle training on the isometric cervical extension strength (ICES) and cross-sectional area (CSA) of the neck extensor muscles.

The subjects which were examined consisted of 22 male college judo athletes. Each was assigned to one of three groups: shrug and upright rowing training (SU); shrug, upright rowing and dynamic neck muscle training (COM); and control (CONT). The SU and COM groups trained 3 days per week for 9 weeks. The ICES and CSA of the neck muscles were measured before and after muscle training.

The ICES of the COM group showed a significant increase after training. For the CSA, although a significant increase was only found in a superficial area of the neck extensor muscles in the $\mathrm{SU}$ group, the COM group showed significant increases in all areas.

This study determined that combined neck muscle training is effective for developing the neck extensor muscles.
\end{abstract}

(Jpn. J. Phys. Fitness Sports Med. 2006, 55 Suppl : S1 S6)

key word : Resistance training, neck extension, muscle strength, cross-sectional area

\section{Introduction}

Neck injuries frequently occur in contact sports such as American football, rugby, wrestling and judo $^{1)}$, and thus the prevention of these injuries is important which can be achieved by the development of neck muscle strength ${ }^{2}$. Brzycki ${ }^{2)}$ reported that strong and thick neck muscles are extremely important for protecting the cervical area from traumatic injuries in such contact sports as football, wrestling, boxing, rugby, soccer and judo. Also, Tsuyama et al. ${ }^{3)}$ showed that isometric cervical extension strength (ICES) and the cross-sectional area (CSA) of neck extensor muscles are significantly increased after dynamic neck muscle training by only using cervical extension machine (CEM). In particular, in creased CSA is larger in the deepest area than in the superficial area. However, to further strengthen the neck muscles, development of the muscle in the superficial layer as well as in the deep layer is re quired, for which no study has been carried out.

The purpose of this study was to examine the effects of two kinds of neck muscles training on the ICES and CSA of the neck extensor muscles.

\section{Method \\ Subjects}

Twenty-two male subjects who belonged to the judo club at N. University in Japan participated in this study. They had no previous symptoms in their

\footnotetext{
${ }^{1)}$ Growth and development, Nippon Sport Science University, 1221-1 Kamoshida-cho, Aoba-ku, Yokohama, Kanagawa, 227-0033, JAPAN

${ }^{2)}$ Sport methodology (judo), Nippon Sport Science University, 7-1-1 Fukasawa, Setagaya-ku, Tokyo, 158-8508, JAPAN

3) Exercise physiology, Nippon Sport Science University, 7-1-1 Fukasawa, Setagaya-ku, Tokyo, 158-8508, JAPAN

${ }^{4)}$ Graduate school of health and sport science, Nippon Sport Science University, 7-1-1 Fukasawa, Setagaya-ku, Tokyo, 158-8508, JAPAN
} 
cervical area. The purposes and procedures associated with participation in the study were explained to all subjects and informed consent was obtained from everyone prior to starting the study. The study was approved by the Ethics Committee of the Nippon Sport Science University.

\section{Strength testing}

The subjects were seated in the CEM, and the position of the seat was adjusted for each subject. The shoulder harness prevented any movement of the torso or pelvis. Also, the mass of the head was counterbalanced to eliminate the influence of gravity. The ICES was measured at eight angles, $126^{\circ}, 108^{\circ}$, $90^{\circ}, 72^{\circ}, 54^{\circ}, 36^{\circ}, 18^{\circ}$ and $0^{\circ}$ using the CEM (MedX Inc. USA). The measurement of the ICES began at $126^{\circ}$ of cervical flexion and thereafter progressed to $0^{\circ}$. These strength testing procedures followed the protocol of Leggett et al. ${ }^{4)}$ and Tsuyama et al. ${ }^{5)}$.

\section{Resistance training}

The subjects $(n=22)$ were ranked by peak ICES and randomly stratified into one of three groups: Control (CONT) Group ( $n=7$ ); Shrug and Upright Rowing (SU) Group ( $\mathrm{n}=7$ ); and Combined (COM) Group $(n=8)$. No significant difference was observed regarding age, height, body weight (BW) and the peak ICES value at pre-training among the three groups (data not shown). The subjects in the CONT group only practiced judo as usual without additional training, while the subjects in the SU group performed shrug and upright rowing training, and the subjects in the COM group performed shrug, upright rowing training and dynamic neck muscle training using the CEM (Fig. 1). Dynamic neck muscle training using the CEM were performed 3 days per week, 3 sets per day, 10 repetitions per set for 9 weeks. The training load used for each subject was calculated from $60 \%$ of the maximal isometric torque of the cervical extension measured by the CEM during pre-training. When 12 or more repetitions could be achieved in the final set, the training load was increased by $5 \%$. In addition, shrug and upright rowing training was performed 3 days per week, 5 sets per day, 10 repetitions per set for 9 weeks. The training load was $70 \% 1 \mathrm{RM}$ of the load, and was confirmed every four weeks.

\section{Magnetic resonance imaging}

The neck muscle CSA was determined for pretraining and post-training using a magnetic resonance imaging (MRI) device manufactured by Hitachi Inc. in Japan. CSA measurements were performed on the subjects of the COM and SU groups, but CSA

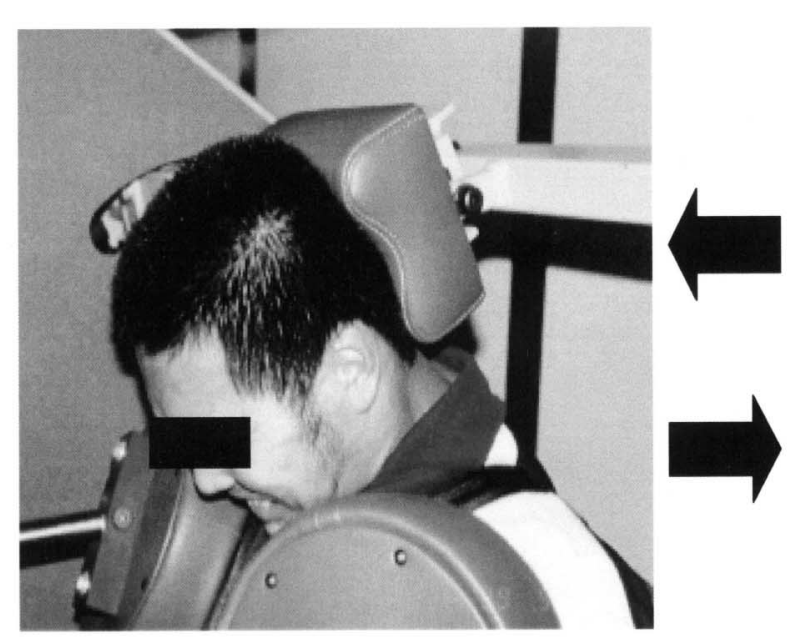

Neck flexed position

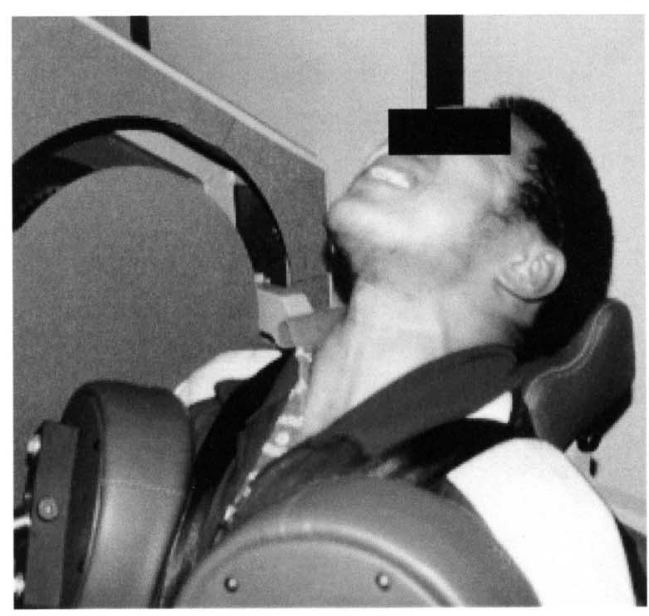

Neck extended position

Fig. 1. Dynamic neck muscle training using the cervical extension machine (CEM). This training was performed from the neck flexed position to the neck extended position. 
measurements were not taken for the CONT group. The areas of B1 to B6 were defined as the cervical extensor muscles: B1 and B2, semispinalis capitis, splenius capitis and cervicis, levator scapulae, longissimus capitis and cervicis, and scalenus; B3 and $\mathrm{B} 4$, rotator, multifidus, and semispinalis cervicis ; and $\mathrm{B} 5$ and $\mathrm{B} 6$, trapezius. In addition, the neck extensor muscles (B1 to B6) at $\mathrm{C} 4-\mathrm{C} 5$ were divided into three areas, $\mathrm{B} 1+\mathrm{B} 2, \mathrm{~B} 3+\mathrm{B} 4$, and $\mathrm{B} 5+\mathrm{B} 6^{3,5)}$, and the CSA of each area was analyzed. We confirmed that the CSA of the neck muscles at C4-C5 mainly represented the neck muscle strength as reported in a previous study ${ }^{6}$. The reliability of the neck muscle CSA determination was confirmed in our previous study ${ }^{3,5)}$.

\section{Statistics}

Differences among the three groups in terms of age, height, BW and maximal ICES at pre-training were analyzed using ANOVA, and the changes in the ICES and the CSA within each group were analyzed using the paired Student's t-test. The level of significance was set at $\mathrm{P}<0.05$.

\section{Results}

There were no significant differences for the ICES in the CONT and SU groups (Fig. 2). However, in the COM group the ICES was increased from 3.8 to $48.0 \%$, and significant increases were observed at 0 , 18, 72 and 90 degrees (Fig. 2).

In the $\mathrm{SU}$ group, the CSA values were increased by $5.0 \%$ in the $\mathrm{B} 1+\mathrm{B} 2$ and $22.4 \%$ in the $\mathrm{B} 5+\mathrm{B} 6$ and significant increases were observed, but there was no significant gain in the area of B3+B4, which is located in the deepest area (Fig. 3). In the COM group, the CSA values for all areas were significantly increased after training (Fig. 3). The rate of CSA increment was $4.9 \%$ at the $\mathrm{B} 1+\mathrm{B} 2,12.1 \%$ at the $\mathrm{B} 3$ $+\mathrm{B} 4$ and $17.4 \%$ at the $\mathrm{B} 5+\mathrm{B} 6$ in the COM group (Fig. 3).

\section{Discussion}

Resistance training is effective for developing
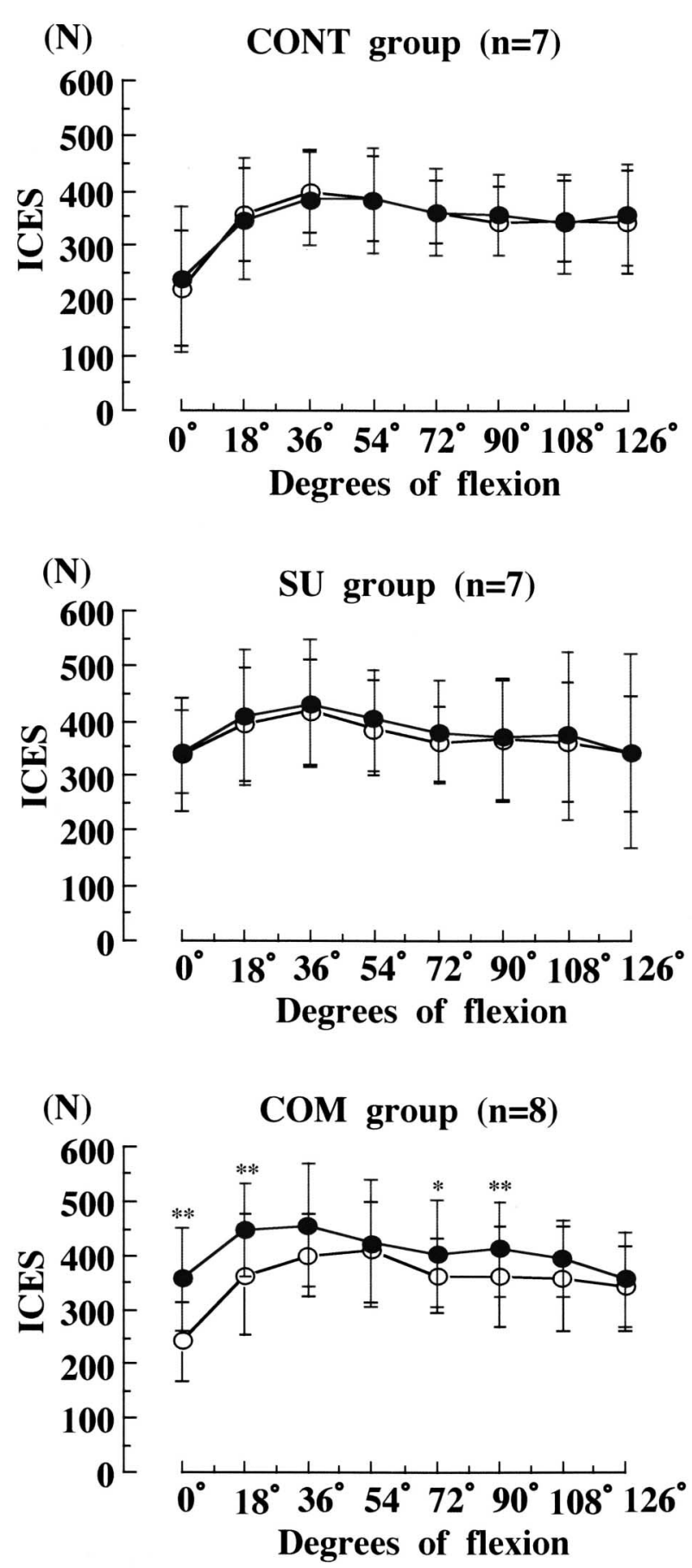

Fig. 2. Comparisons of isometric cervical extension strength (ICES) pre-training and post-training values in each group.

The opened circles are values for pre-training and the closed circles are values for post-training. $\left({ }^{* *}: \mathrm{p}<0.01\right.$, $*: \mathrm{p}<0.05)$.

muscle strength ${ }^{2 \sim 4)}$. Many studies on resistance training have been performed for muscles of the upper and lower limbs ${ }^{7}$. However, resistance training for the neck muscles has yet to be thoroughly stu- 

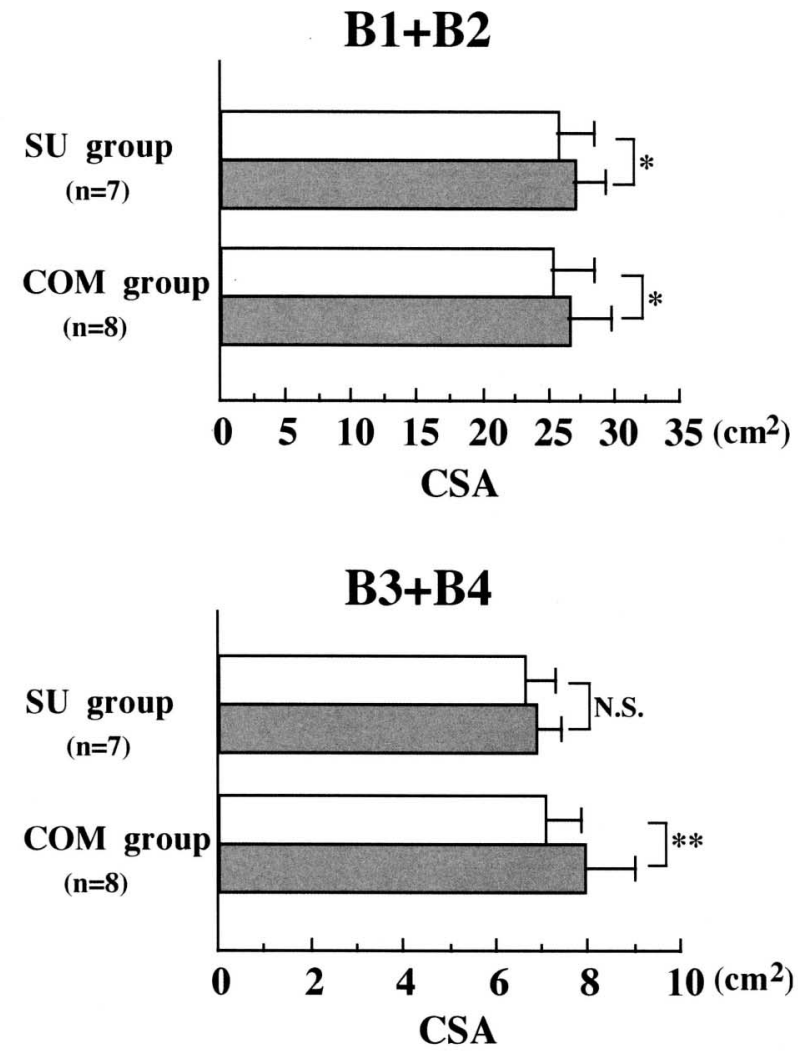

B5+B6

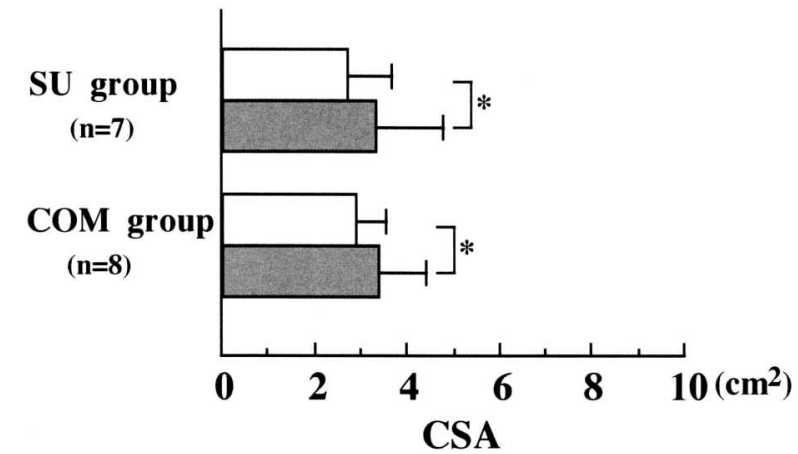

Fig. 3. Comparisons of cross-sectional area (CSA) values in three groups of neck extensor muscles between pre-training and post-training.

The opened bars are values for pre-training and the closed bars are values for post-training. B1 and B2, semispinalis capitis, splenius capitis and cervicis, levator scapulae, longissimus capitis and cervicis and scalenus ; $\mathrm{B} 3$ and B4, rotator, multifidus and semispinalis cervicis ; and B5 and B6, trapezius. $\left({ }^{* *}: \mathrm{p}<0.01,{ }^{*}: \mathrm{p}<0.05\right.$, N.S. : not significant).

died, but has been performed using a few methods ${ }^{8}$ 10), one of which is carried out using the $\mathrm{CEM}^{3,4}$, 10). Pollock et al. ${ }^{10)}$ reported that the increase in the ICES ranges from 17.2 to $32.8 \%$ when resistance training of the neck muscles using the CEM is per- formed 1 set per day and 2 days per week with an $80 \%$ training load for 12 weeks. In this study, the ICES in the COM group was significantly increased after training, but the ICES in the SU and CONT groups was not significantly increased. These results showed that resistance training which involves neck extension and flexion movements such as that with the CEM is required to increase neck muscle strength during a short period.

Conley et al. ${ }^{9)}$ reported a $13 \%$ increase in the total neck muscle CSA after resistance training of the neck muscles for 12 weeks. In particular, the in crease in the CSA was larger in the splenius capitis, semispinalis capitis, semispinalis cervicis and multifidus muscles, which are located in the deep layer of the neck muscles.

In this study, the CSA in the COM group was significantly increased for all areas of the neck extensor muscles, but the CSA in the SU group was only significantly increased in the superficial area except for the deepest area. In our previous study ${ }^{3)}$, we in vestigated the effects of neck muscle training only using the CEM on ICES and CSA of the neck extensor muscles. We observed a significant gain in the CSA in the deep layer of the neck extensor muscles, although there was no significant gain in the superficial layer. Conley et al. ${ }^{11)}$ showed that the semispinalis capitis, semispinalis cervicis and multifidus, and splenius capitis muscles in the deep layer of the neck muscles are markedly used during head extension exercises, while the trapezius muscle in the superficial layer of the neck extensor muscles is used much less. Based on these results, we considered that resistance training which utilizes neck extension and flexion movements such as that with the CEM mainly develops the deep layer of the neck extensor muscles, while resistance training which in volves elevating the scapula such as that for shrug and upright rowing training mainly develops the superficial layer of the neck extensor muscles. Therefore, to increase all areas of the neck extensor muscles, resistance training involving neck extension and flexion movements such as that with the CEM must 
be performed along with shrug and upright rowing training.

In general, when resistance training is performed, muscle strength is increased at first, and after that the CSA is increased ${ }^{12)}$. However, in the SU group, the ICES was not significantly increased after training, but the CSA in the superficial layer was significantly increased. The rate of contribution to the neck muscle strength is different from each area, $\mathrm{B} 1$ $+\mathrm{B} 2, \mathrm{~B} 3+\mathrm{B} 4$, and B5 + B6, of the neck extensor muscles. In our previous study ${ }^{13)}$, the ICES and CSA values were compared between wrestlers and weight lifters, and we found that the ICES values of the wrestlers were significantly higher than those of the weight lifters. In addition, the CSA values of the wrestlers in the deep layer of the neck extensor muscles were significantly higher than those of the weight lifters, although the CSA value of the weight lifters was significantly higher in the superficial layer, which contains the trapezius muscles, than that of the wrestlers. Conley et al. ${ }^{11)}$ showed that the neck muscles in the deep layer of the neck extensor muscles are more activated during head extension exercises, while the trapezius muscle in the superficial layer is not. Based on these results, it was inferred that the rate of contribution to the ICES is higher in the deep layer than in the superficial layer. Therefore, the ICES was not increased after training in the SU group although the CSA in the superficial layer was significantly increased.

\section{References}

1) Katoh, S., Shingu, H., Ikata, T. \& Iwatsubo, E. Sportsrelated spinal cord injury in Japan (from the nationwide spinal cord injury registry between 1990 and 1992). Spinal Cord,(1996), 34, 416-421.

2) Brzycki, M. Strengthening the neck: Reducing risk of cervical injury. Athletic J.,(1985), 65, 40-48.

3) Tsuyama, K., Yamamoto, Y., Nakazato, K. \& Nakajima, H. Effects of dynamic neck muscle training on strength and cross-sectional area of neck muscles in judo athletes (in Japanese). Jpn. J. Phys. Fitness Sports Med.,(2005), 54, 249-258.

4) Leggett, S. H., Graves, J. E., Pollock, M. L., Shank, M., Carpenter, D. M., Holmes, B. \& Fulton, M. Quantitative assessment and training of isometric cervical extension strength. Am. J. Sports Med.,(1991), 19, 653659.

5) Tsuyama, K., Yamamoto, Y., Fujimoto, H., Adachi, T., Nakazato, K., \& Nakajima, H. Comparison of the isometric cervical extension strength and a crosssectional area of neck extensor muscles in college wrestlers and judo athletes. Eur. J. Appl. Physiol., (2001), 84, 487-491.

6) Tsuyama, K., Yamamoto, Y., Fujimoto, H., Nakazato, K., Fujishiro, H. \& Nakajima, H. Relationship between isometric cervical extension strength based on a cervical extension machine and the cross-sectional area of neck muscles (in Japanese). Jpn. J. Phys. Fitness Sports Med.,(2000), 49, 193-202.

7) Ikai, M. \& Fukunaga, T. A study on training effect on strength per unit cross-sectional area of muscle by means of ultrasonic measurement. Int. Z. Agnew Physiol,(1970), 28, 173-180.

8) Stump, J., Rash, G., Semon, J., Christian, W. \& Miller, $\mathrm{K}$. A comparison of two modes of cervical exercise in adolescent male athletes. J. Manipulative Physiol. Ther.,(1993), 16, 155-160.

$9)$ Conley, M. S., Stone, M. H., Nimmons, M. \& Dudley, G. A. Specificity of resistance training responses in neck muscle size and strength. Eur. J. Appl. Physiol.,(1997), 75, 443-448.

10) Pollock, M. L., Graves, J. E., Bamman, M. M., Legget, S. H., Carpenter, D. M., Carr, C., Cirulli, J., Matkozich, J. \& Fulton, M. Frequency and volume of resistance training: effect on cervical extension strength. Arch. Phys. Med. Rehabil.,(1993), 74, 1080-1086.

11) Conley, M. S., Meyer , R. A., Bloomberg, J. J., Feeback, D. L. \& Dudley, G. A. Noninvasive analysis of human neck muscle function. Spine, (1995), 20, 2505-2512.

12) Moritani, T. \& deVries, H. A. Neural factors versus hypertrophy in the time course of muscle strength gain. Am. J. Phys. Med.,(1979), 58, 115-130.

13) Tsuyama, K., Sekiguchi, O., Hosotani, J., Fujimoto, H., Yamato, M., Kiyota, H., Takeda, M., Hamano, M. \& Nakajima, H. Comparison of the isometric cervical extension strength and a cross-sectional area of neck extensor muscles in college wrestlers and weight lifters (in Japanese). Jpn. J. Phys. Fitness Sports Med., (2002), 51, 768 . 